\title{
UTILIZAÇÃO DO MÉTODO MULTICRITÉRIO TODIM-FSE PARA CLASSIFICAÇÃO DE BASE LOGÍSTICA DE BRIGADA ${ }^{1}$
}

\author{
Cleidinei Augusto da Silva \\ Academia Militar das Agulhas Negras (AMAN), Rodovia Presidente Dutra, Km 306 - \\ Resende, RJ, cleidinei@ hotmail.com
}

Luís Alberto Duncan Rangel, Tiago Araújo Neves

Escola de Engenharia Industrial Metalúrgica de Volta Redonda-UFF (EEIMVR)

Av dos Trabalhadores, 420 - Vila Santa Cecília - Volta Redonda, RJ, duncan@metal.eeimvr.uff.br, tneves@id.uff.br

Luiz Flavio Autran Monteiro Gomes

Ibmec/RJ, Av Presidente Wilson 118, $11^{\circ}$ andar, Rio de Janeiro, RJ

autran@ibmecrj.br

\begin{abstract}
RESUMO
Um dos temas mais relevantes relacionados ao sucesso de uma força militar é o apoio logístico. No processo decisório em questão foi utilizado o método multicritério TODIM-FSE. Um conjunto de instalações logísticas, como armazenagem, oficinas, hospitais e outros são obrigados a atender as múltiplas demandas de uma operação militar. Estas instalações devem ser localizadas dentro de uma zona conhecida como Base Logística de Brigada. O processo de tomada de decisão a respeito desta área é consideravelmente complexa, não só por causa do possível número elevado de áreas candidatas, mas também por causa dos critérios de conflito que podem ser enfrentados durante esta seleção. Os critérios podem por vezes, ser subjetivo ou dependente em diferentes escalas de valor. Este trabalho apresenta um método analítico de apoio multicritério à tomada de decisão para classificação da base, seguindo regras estabelecidas na doutrina militar do Exército Brasileiro. A conclusão do trabalho sugere oportunidades de melhoria na tomada de decisões logísticas, que consequentemente pode implicar em maior efetividade na gestão dos recursos escassos.
\end{abstract}

Palavras-Chave: Apoio multicritério à decisão, logística militar, método TODIM-FSE, classificação.

\begin{abstract}
Logistic support is one of the most important aspects of military operations. A set of logistic facilities such as storages, hospitals and mechanic shops are needed in order to handle de demands of such operations. These facilities must be placed inside a zone known as "Base Logística de Brigada". The decisions involving this area are quite complex not only because the high number of candidate areas but also due to the conflicting criteria involved in the selection. The criteria may be subjective or dependent in different scales. This paper proposes the use of the analytic multi-criteria method TODIM-FSE for supporting in bases classification using rules established by the Brazilian Army. Our conclusion is that there are opportunities to improve logistic decisions and, consequently, improve resources management.
\end{abstract}

Keywords: Multicriteria Decision Aid, military logistic, TODIM-FSE method, rank.

\footnotetext{
${ }^{1}$ Agradecimentos: os autores agradecem ao Curso de Intendência da Academia Militar das Agulhas Negras (AMAN) - Resende/RJ e à Divisão de Doutrina e Pesquisa do Centro de Doutrina do Exército - Brasília/DF.
} 


\section{INTRODUÇÃO}

A tomada de decisão em ambientes militares está cada vez mais complexa influenciada por diversos fatores, recursos limitados, variáveis tangíveis e intangíveis no processo decisório e a velocidade de interação entre eles, alterando-se continuamente devido aos cenários de risco e incerteza.

Utilizando-se do conceito de "Logística na Medida Certa" (Ministério da Defesa, 2014), depreende-se neste sentido a necessidade de designar com a maior qualidade possível os diversos locais onde ocorrerão as atividades de suprimentos e logística em operações de guerra e não-guerra.

Em pesquisa operacional, os métodos de Apoio Multicritério à Decisão (AMD) são aplicados em casos onde na presença de múltiplos critérios, busca-se selecionar, ordenar, classificar ou descrever alternativas, dependendo da problemática abordada. Os métodos do AMD podem ser classificados dentro de duas grandes Escolas: a Americana e a Francesa (ou Européia) (Belton, Stewart, 2002).

O método TODIM-FSE (Passos et al, 2013) contém elementos técnicos dessas duas escolas, denominados métodos híbridos. Este artigo objetiva aplicar o método TODIM-FSE para classificar as candidatas a áreas que possuam o desempenho pertinente em termos do preconizado pela doutrina militar terrestre brasileira.

O artigo está organizado da seguinte forma: na seção 2 aborda-se a aspectos teóricos de logística no Exército Brasileiro (EB), fundamental para a compreensão dos critérios adotados e na seção 3 é apresentado o método TODIM-FSE. Nas seções seguintes apresentam-se o estudo de caso hipotético, as discussões e as conclusões.

\section{ASPECTOS LOGÍSTICOS DA FORÇA TERRESTRE BRASILEIRA}

Na doutrina do Exército Brasileiro (Ministério da Defesa, 2014), a Base Logística de Brigada (BLB) é a área onde são desdobrados os meios orgânicos dos Batalhões Logísticos e outros recursos específicos necessários ao apoio a uma grande unidade. Sua organização é modular e fundamentada em meios dotados de mobilidade tática, de modo a possibilitar o apoio logístico às operações e assegurar certo grau de autonomia à força apoiada. Tem como finalidade executar o apoio logístico às forças integrantes de um grande comando operacional e, conforme determinado, a outras forças e à população civil.

Uma das fases fundamentais do ciclo logístico é a distribuição que consiste em fazer chegar aos usuários, oportuna e efetivamente, todos os recursos fixados pela determinação das necessidades. A distribuição engloba um sistema de pessoal, instalações, técnicas e procedimentos, visando a receber, acondicionar, movimentar, entregar e controlar o fluxo da cadeia logística entre o ponto de recepção e o ponto de destino. A flexibilidade e a adaptabilidade aplicadas ao sistema de distribuição asseguram a melhor utilização possível dos meios de transporte disponíveis e reduzem ao mínimo indispensável os percursos, os transbordos e os manuseios dos recursos. É neste aspecto que a adequada localização das instalações militares seja efetivada para obter-se a melhor estrutura do apoio logístico as operações.

A BLB é localizada na Zona de Combate (ZC), de acordo com os planejamentos operacionais e as necessidades logísticas decorrentes. Situa-se na área de retaguarda do escalão considerado, podendo-se admitir a exceção. Para a localização da área de apoio logístico de divisão de exército ou de brigada devem ser considerados os fatores que se seguem: manobra tática do escalão considerado; as características das prováveis áreas de desdobramento (terreno); as condições de segurança para a prestação do apoio; a situação logística existente; e, outros fatores. A Figura 1 apresenta os principais fatores preconizados na doutrina militar terrestre. 


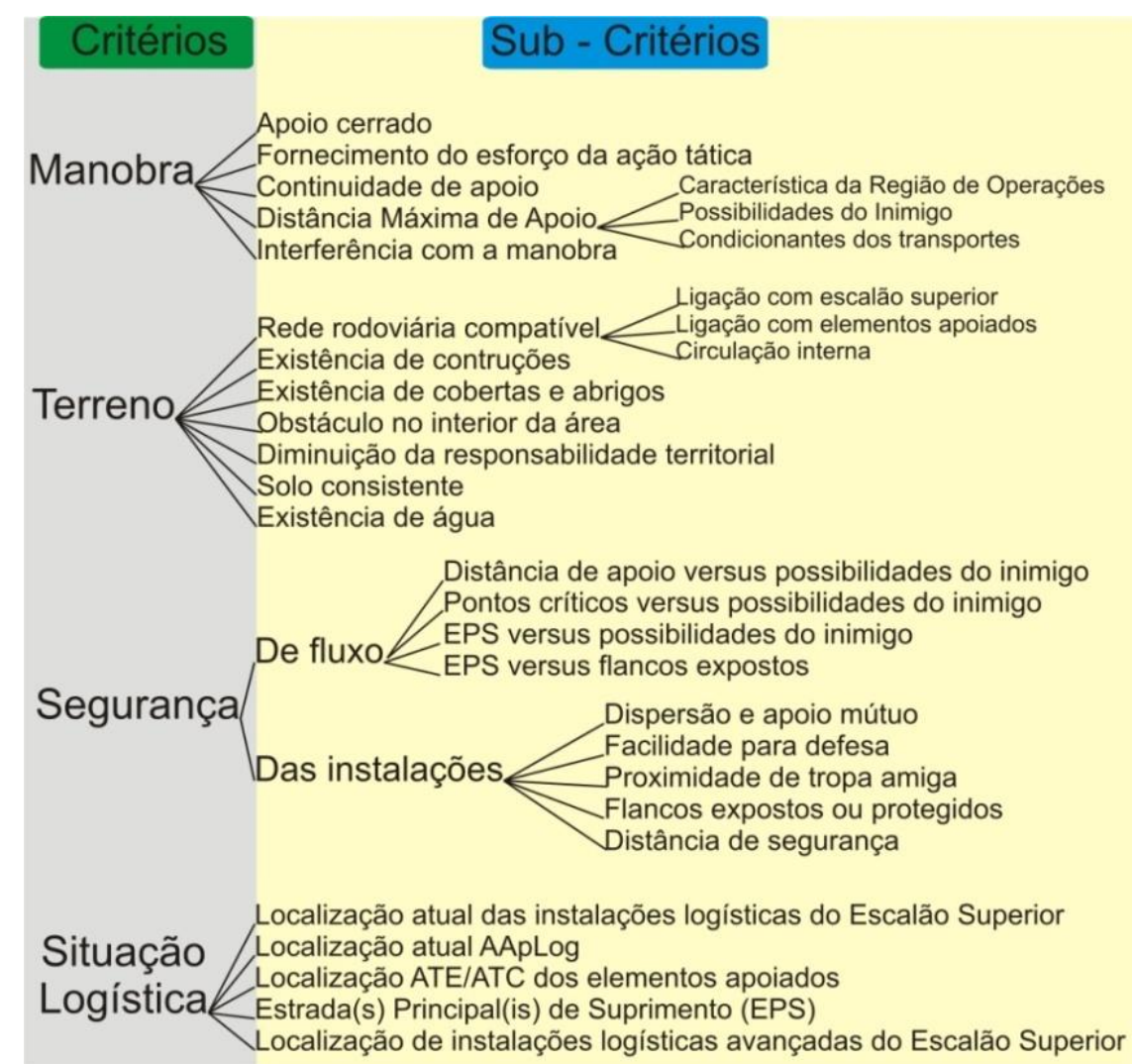

Figura 1 - Fatores determinantes para escolha de localização de Base Logística (Ministério da Defesa, 2003)

Baseado no manual de campanha Apoio Logístico na Divisão de Exército e na Brigada (Ministério da Defesa, 2003) tem-se as características de alguns subfatores que compõem os critérios supracitados neste artigo:

- Do fator manobra - Distância máxima de apoio (DMA) - É a maior distância, medida por estrada, admitida entre a área de apoio logístico e as áreas de trens de estacionamento das unidades de combate (ATE) ou áreas de trens das unidades de apoio ao combate (AT). Essa distância, expressa em quilômetros, baseia-se no fato de que as atividades de suprimento se desenvolvem no período noturno, espaço de tempo de que dispõem as viaturas para realizar o percurso de ida e volta entre as instalações de suprimento e os trens dos elementos apoiados. Seu valor resulta da multiplicação do número de horas disponíveis para o deslocamento pela velocidade média estimada;

- Do fator terreno - Rede rodoviária compatível - Esse aspecto trata da capacidade de tráfego, quando se refere às vias utilizadas nas ligações externas, que assegurem ligações com o escalão superior e elementos apoiados, e da disposição da malha viária, quando se refere à circulação no interior da área. Daí sua abordagem em três enfoques: ligação com o escalão superior, Ligação com os elementos apoiados e circulação interna;

- Do fator segurança - Segurança das instalações - Dispersão e apoio mútuo, Facilidade para a defesa, Proximidade de tropa amiga, Flancos expostos ou protegidos, Distância de segurança;

- Do fator situação logística - Estrada(s) principal(is) de suprimento em uso e as previstas para serem usadas no prosseguimento das ações.

\section{TOMADA DE DECISÃO MULTICRITÉRIO}

Dada a complexidade de decisão da definição do local que melhor atenda às necessidades ilimitadas e os recursos escassos, apresenta-se uma metodologia que favorece a escolha oportuna diante das diversas variáveis existentes para o desdobramento logísticooperacional de uma tropa. 
O método TODIM-FSE (Passos et al, 2013) é uma metodologia de apoio à decisão multicritério de classificação de alternativas discretas que utiliza como referência o método TODIM (Gomes, Rangel, Maranhão, 2009; Gomes e Rangel, 2009; Gomes e Lima, 1992; Rangel, Gomes, Cardoso, 2011), fundamentado na Prospect Theory, e a Avaliação Sintética Fuzzy (Lu, Lo, Hu, 1999; Onkal-Engin e Demir, 2004; Chang, Chen, Ning, 2001; Sadiq et al, 2004; Kuo e Chen, 2006).

O método TODIM é um método multicritério de ordenação de alternativas sedimentado na literatura científica. Segundo Passos et al (2013), o método FSE apesar de não ser conhecido como um método multicritério já foi utilizado desta forma.

O TODIM-FSE vem contribuir como uma opção para as aplicações típicas de classificação de alternativas utilizando múltiplos critérios.

A Prospect Theory pertence ao campo da psicologia cognitiva e trata da maneira como os seres humanos decidem em situações que envolvem riscos. Através de um conjunto de experimentos realizados na década de 1970, Daniel Kahneman e Amos Tversky (1979) conseguiram mostrar alguns comportamentos dos seres humanos, até então desconhecidos. Perceberam que nas situações que envolvem ganhos, seres humanos costumam ser mais conservadores em relação ao risco e, em situações que envolvem perdas, mostram-se mais propensos ao risco. Isto é, quando se estabelece uma situação em que se pode ganhar, preferem um ganho menor, porém certo, a se arriscar por ganhos maiores e incertos. Em situações que envolvem perdas, as pessoas preferem se arriscar a perder mais, porém, com a possibilidade de nada perderem, a ter uma perda menor, porém, certa. Adicionalmente, foi percebido que as situações envolvendo perdas costumavam ser muito mais relevantes e impactantes que situações envolvendo ganhos.

Para representar graficamente este comportamento, foi mostrada em artigo (Kahneman e Tversky, 1979) uma função de valor que ilustra o comportamento descrito no parágrafo anterior, que é relevante para entender as equações utilizadas no método TODIM-FSE. A Figura 2 ilustra este comportamento.

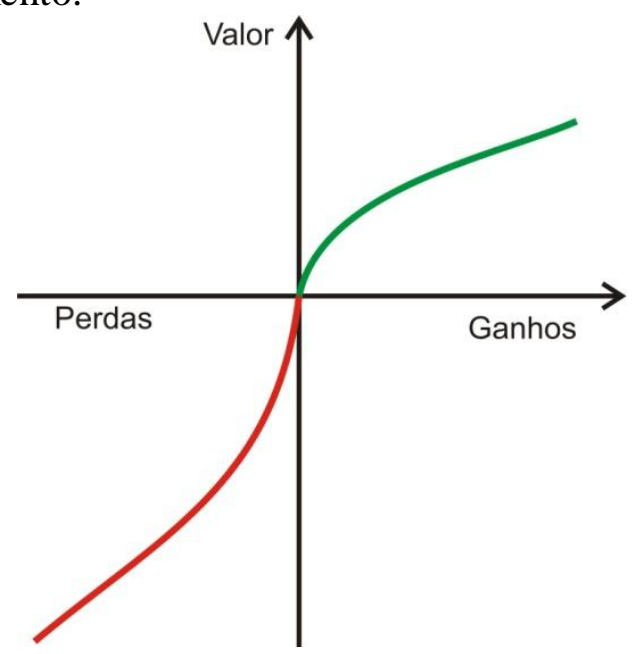

Figura 2 - Função de Valor da Teoria dos Prospectos (Adaptada de Kahneman e Tversky, 1979).

A partir da incorporação dessa função de valor, permite-se estabelecer uma medida quantitativa da satisfação das pessoas, inserindo ao modelo a característica de aversão e propensão ao risco, natural dos seres humanos.

Citando Passos et al. (2013), para facilitar a compreensão e utilização do método ele será descrito em etapas seguindo o exemplo de Goodwin e Wright (2004). As fases abaixo não precisarão seguir rigorosamente a sequência proposta:

Fase 1: Definição dos decisores e analistas de decisão

Os tomadores de decisão são as pessoas que vão fazer julgamentos sobre o problema de decisão. Os analistas de decisão são os especialistas em processos de apoio à decisão e métodos. 
Fase 2: Análise e estruturação do problema decisório

Nesta etapa é analisado o problema em questão e discutido minuciosamente, para que haja certeza de que o problema relevante está sendo abordado.

Fase 3: Definição dos critérios relevantes para o problema

Nesta fase ocorre o início da construção do modelo decisório. Os critérios devem ser filtrados, fundidos ou eliminados de forma que sejam respeitadas as recomendações de Keeney e Raiffa (1976) para a construção de um bom conjunto de critérios. Sendo desejáveis dos critérios selecionados as seguintes características: operacionalidade, decomponibilidade, tamanho mínimo, completude e não redundância.

\section{Fase 4: Definição de categorias e funções de contribuição}

Após definida a quantidade $k$ de categorias devem ser definidas as contribuições $\mu$ que cada critério fornece para que uma alternativa se classifique dentro de determinada categoria. Conforme cita Passos et al. (2013), o conceito de contribuição, até o melhor do nosso conhecimento, é inovador no sentido que está sendo dado neste método. Os valores de contribuição deverão variar entre 0 (zero) e 1 (um) continuamente, sendo que o valor 1 (um) indica que o critério contribui ao máximo para que uma alternativa se classifique dentro de determinada categoria. $\mathrm{O}$ valor 0 (zero) indica que o critério não contribui para que a alternativa se classifique dentro de determinada categoria. Além disso, são permitidos valores intermediários de contribuição.

Para cada tipo de critério, qualitativo ou quantitativo, os valores de contribuição serão definidos de forma diferente. Se o critério $i$ for qualitativo, espera-se que a sua avaliação $\gamma$ se dê através de uma escala com valores discretos. Para cada valor verbal da escala serão definidos valores de contribuição para cada uma das categorias na forma de tabelas de contribuição, conforme mostrado na Tabela 1.

\begin{tabular}{cccccc}
\hline \multirow{2}{*}{ Avaliação } & \multicolumn{5}{c}{ Categorias } \\
& $\mathrm{Cat}_{1}$ & $\mathrm{Cat}_{2}$ & $\ldots \ldots$ & Cat $_{\mathrm{k}-1}$ & Cat $_{\mathrm{k}}$ \\
\hline$\gamma_{1}$ & $\mu_{11}$ & $\mu_{12}$ & $\ldots \ldots$ & $\mu_{1 \mathrm{k}-1}$ & $\mu_{1 \mathrm{k}}$ \\
$\gamma_{2}$ & $\mu_{21}$ & $\mu_{22}$ & $\ldots \ldots$ & $\mu_{2 \mathrm{k}-1}$ & $\mu_{2 \mathrm{k}}$ \\
$\ldots$ & $\ldots$. & $\ldots$. & $\ldots \ldots$ & $\ldots \ldots$ & $\ldots$. \\
$\gamma_{\mathrm{m}}$ & $\mu_{\mathrm{m} 1}$ & $\mu_{\mathrm{m} 2}$ & $\ldots \ldots$ & $\mu_{\mathrm{mk}-1}$ & $\mu_{\mathrm{mk}}$ \\
\hline
\end{tabular}

Tabela 1: Tabela de contribuições para o critério i, qualitativo, (Adaptada de Passos et al, 2013)

Para cada possível avaliação $\gamma_{i}$ atribuída para esse critério será definido um conjunto de contribuições, representado pela linha correspondente na Tabela 1.

Se o critério $j$ for quantitativo ele poderá assumir valores contínuos. Neste caso as contribuições serão representadas através de funções de contribuição, que serão parecidas com conjuntos fuzzy, na forma e na maneira de construção. A Figura 3 ilustra um exemplo de funções de contribuição descritas com funções sigmoidais, para três categorias.

Após definidas as tabelas de contribuição ou funções de contribuição para cada critério será possível agrupar o primeiro conjunto de dados importante para o modelo, aqui chamado de "tabela de contribuições agrupadas dos critérios". Cada linha dessa tabela é obtida a partir da avaliação realizada para a alternativa a luz de cada critério. Para os critérios qualitativos representam uma linha da Tabela 1. Para critérios quantitativos representam o valor da função de contribuição associado ao valor quantitativo atribuído para o critério $j$. A Tabela 2 ilustra um exemplo. 


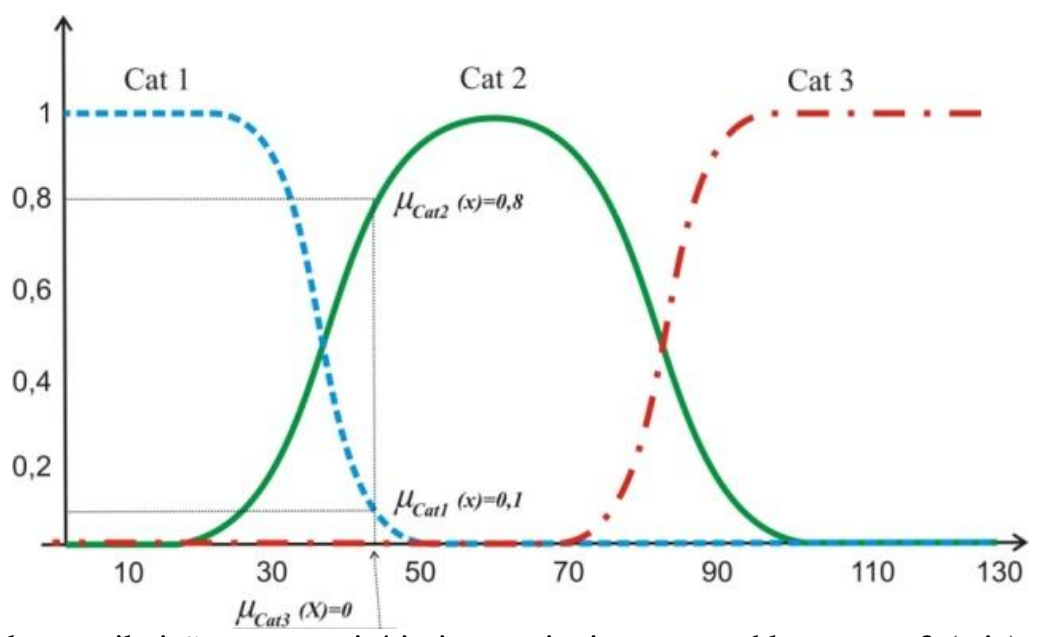

Figura 3: Funções de contribuição para o critério j, quantitativo, em problema com 3 (três) categorias. No caso em que o critério assume o valor 45 é possível obter o seguinte vetor de contribuições associado a cada uma das categorias $[0,1 \quad 0,80]$. Assim, quando o critério j assume o valor 45 ele está contribuindo com 0,1 para que a alternativa se classifique na primeira categoria; 0,8 para que a alternativa se classifique na segunda categoria e zero para que a alternativa se classifique na categoria 3, (Adaptada de Passos et al, 2013).

\begin{tabular}{cccccc}
\hline \multirow{2}{*}{ Critérios } & \multicolumn{5}{c}{ Categorias } \\
& Cat $_{1}$ & Cat $_{2}$ & $\ldots \ldots$ & Cat $_{\mathrm{k}-1}$ & Cat $_{\mathrm{k}}$ \\
\hline $\mathrm{C}_{1}$ & $\mu_{11}$ & $\mu_{12}$ & $\ldots \ldots$ & $\mu_{1 \mathrm{k}-1}$ & $\mu_{1 \mathrm{k}}$ \\
$\mathrm{C}_{2}$ & $\mu_{21}$ & $\mu_{22}$ & $\ldots \ldots$ & $\mu_{2 \mathrm{k}-1}$ & $\mu_{2 \mathrm{k}}$ \\
$\ldots$ & $\ldots$. & $\ldots$. & $\ldots \ldots$ & $\ldots \ldots$ & $\ldots$. \\
$\mathrm{C}_{\mathrm{m}}$ & $\mu_{\mathrm{m} 1}$ & $\mu_{\mathrm{m} 2}$ & $\ldots \ldots$ & $\mu_{\mathrm{mk}-1}$ & $\mu_{\mathrm{mk}}$ \\
\hline
\end{tabular}

Tabela 2: Tabela de contribuições agrupadas dos critérios, (Adaptada de Passos $e$ t al, 2013)

Cada linha dessa tabela é formada pelas contribuições associadas a avaliação feita para cada critério. Para um critério qualitativo é extraída da sua tabela de contribuição a linha associada à avaliação efetuada. A linha extraída é copiada na linha correspondente da tabela acima. Para um critério quantitativo, o vetor de contribuições obtido deve ser copiado na linha correspondente na tabela acima.

Fase 5: Definição da importância relativa entre os critérios

Os procedimentos para obter os pesos dos critérios são:

a. Preencher uma matriz de comparações paritárias similar a utilizada no AHP (Saaty, 2006), onde nela são inseridos julgamentos extraídos da escala fundamental de Saaty, apresentada na Tabela 3. Obtém-se assim o vetor de pesos para $n$ critérios $\left[\begin{array}{lll}a_{1} & a_{2}, \ldots, a_{a n-1} & a_{n}\end{array}\right]$.

\begin{tabular}{cl}
\hline Escala Numérica & \multicolumn{1}{c}{ Escala Verbal } \\
\hline 1 & Igual importância \\
3 & Importância moderada \\
5 & Importância forte \\
7 & Importância muito forte \\
9 & Importância extrema \\
$2,4,6,8$ & Valores intermediários \\
\hline
\end{tabular}

Tabela 3: Escala fundamental (Saaty, 2006)

b. Para retirar qualquer possível inconsistência existente nestes julgamentos paritários é utilizado o mesmo procedimento proposto no método TODIM. Assim, com o vetor de pesos obtido é construída uma nova matriz,

c. A partir desta matriz é obtido um novo vetor de pesos. Cada uma de suas componentes é calculada através da média aritmética das linhas da matriz acima. Depois disso é feita a normalização de cada desses valores dividindo-os pela soma dessas médias. Esse 
procedimento é feito para evitar os problemas com o cálculo do autovetor da matriz, mencionados por Bana e Costa e Vansnick (2008). O resultado é o vetor de pesos $W$, apresentando na Equação (1), cuja soma das componentes é igual a 1, indicado na Equação (2).

$W=\left[\begin{array}{llllll}\mathrm{w}_{1} & \mathrm{w}_{2} & \mathrm{w}_{3} & \ldots \ldots \ldots . . . & \mathrm{w}_{n-1} & \mathrm{w}_{n}\end{array}\right]$

$\sum_{i=1}^{n} \mathrm{w}_{i}=1$

Fase 6: Classificação de cada alternativa em uma das categorias propostas

Definidos a tabela de contribuições agrupadas dos critérios Tabela 2 e os pesos dos critérios, passa-se a utilizar os trade-offs embutidos nos pesos dos critérios e agregar tudo para encontrar em qual categoria a alternativa possuirá a maior pontuação irá se classificar. Para tal será utilizado às equações do método TODIM.

Conforme Gomes (2004), são construídas $n$ matrizes de dominâncias parciais $\Phi_{c}$, uma para cada critério $c$. Os elementos de cada uma dessas matrizes serão dados pela Equação (3),

$\Phi_{c}\left(\mathrm{cat}_{i}, \mathrm{cat}_{j}\right)=\left\{\begin{array}{cl}\sqrt{\frac{w_{r c}\left(\mu_{i c}-\mu_{j c}\right)}{\sum_{c=1}^{n} w_{r c}}}, & \mu_{i c}-\mu_{j c} \geq 0 \\ -\frac{1}{\theta} \sqrt{\frac{\sum_{c=1}^{n} w_{r c}\left(\mu_{j c}-\mu_{i c}\right)}{w_{r c}}}, & \mu_{i c}-\mu_{j c}<0\end{array}\right.$

Verifica-se que na Equação (3), que $\mu_{i c}$ e $\mu_{j c}$ representam pesos das alternativas $i$ e $j$, respectivamente em relação ao critério $c$. Sendo que as diferenças $\mu_{i c}-\mu_{j c} \geq 0$ e $\mu_{i c}-\mu_{j c}<$ 0 são consideradas como ganhos ou perdas associados à função de valor da Teoria dos Prospectos, conforme representado graficamente na Figura 2.

Se a diferença for positiva (representando ganho de dominância da categoria $i$ frente à categoria $j$ ) o valor do elemento genérico $a_{i j}$ da matriz $\Phi_{c}$ será dado pela primeira função da Equação (3), se a diferença for negativa (sugerindo perda de dominância da contribuição da categoria $i$ frente à categoria $j$ ) o valor deste mesmo elemento $a_{i j}$ será dado pela segunda função da Equação (3).

Os valores $w_{r c}$ representam uma taxa de substituição, ou seja, o peso do critério $c$ dividido pelo peso do critério de referência $r$. O valor $\theta$ é o fator de atenuação de perdas. Diferentes escolhas deste valor levam a diferentes formas da função de valor da Teoria dos Prospectos no quadrante negativo, Figura 1.

Assim, cada matriz $\Phi_{c}$ estará armazenando um conjunto de valores de dominância das categorias com relação a cada critério.

Depois de calculadas as matrizes de dominâncias parciais para cada critério será calculada a matriz de dominâncias $\delta\left(\right.$ cat $_{i}$, cat $\left._{j}\right)$ equacionada como:

$\delta\left(\mathrm{cat}_{i}, \mathrm{cat}_{j}\right)=\sum_{c=1}^{n} \Phi_{c}\left(\mathrm{cat}_{i}, \mathrm{cat}_{j}\right), \forall(i, j)$

Da matriz formada pela equação (4), tem-se que cada elemento soma todas as dominâncias obtidas anteriormente para cada critério. O resultado final será obtido com o cálculo do vetor $X$, que terá como cada um de seus elementos o seguinte valor:

$\xi_{i}=\frac{\sum_{j=1}^{k} \delta\left(\text { cat }_{i}, c a t_{j}\right)-\min \sum_{j=1}^{k} \delta\left(\text { cat }_{i}, c a t_{j}\right)}{\operatorname{máx} \sum_{j=1}^{k} \delta\left(\text { cat }_{i}, c a t_{j}\right)-\min \sum_{j=1}^{k} \delta\left(\text { cat }_{i}, c a t_{j}\right)}$

Ao ser calculado dessa forma o vetor $X$ possuirá sempre uma componente com valor 1, representando a categoria mais adequada para a classificação, outro com valor 0 (zero), representando a categoria menos adequada para a classificação, e outros valores intermediários para as demais categorias.

$\underline{\text { Fase 7: Análise de validação }}$

A análise de validação, neste método, terá grande importância para a criação de um modelo de apoio à decisão de qualidade. Nela, alternativas previamente classificadas dentro de cada uma das categorias propostas, serão usadas como referência para ajustar o modelo criado. Esses ajustes podem ser feitos nos pesos dos critérios, assim como nas tabelas ou funções de contribuições. 


\section{CLASSIFICAÇÃO BLB PELA ABORDAGEM PROPOSTA}

Para mostrar a implementação do TODIM-FSE, um estudo de caso hipotético de classificação de BLB será executado. O objetivo desta avaliação é selecionar área para um desdobramento de tropas de apoio logístico.

Utilizando as informações levantadas pelo Estado Maior de um Comando Militar é feita a classificação dentro de uma entre quatro categorias: excelente, muito adequado, adequado ou pouco adequado. Para efetuar essa avaliação será utilizado quatro subcritérios preconizados na doutrina militar referentes aos fatores: manobra, terreno, segurança e situação logística.

Modelo de Avaliação hipotético para Escolha de Base Logística de Brigada (BLB ou AApLog) (área com dimensões médias de 2000 m x 3000 m)

As alternativas estão limitadas a 3 possíveis áreas de apoio logístico dentro da Zona de Combate (ZC). Na Figura 4, observa-se as áreas que representam as alternativas a serem escolhidas são: 1-Área Alfa (A), 2-Área Bravo (B), 3-Área Charlie (C).

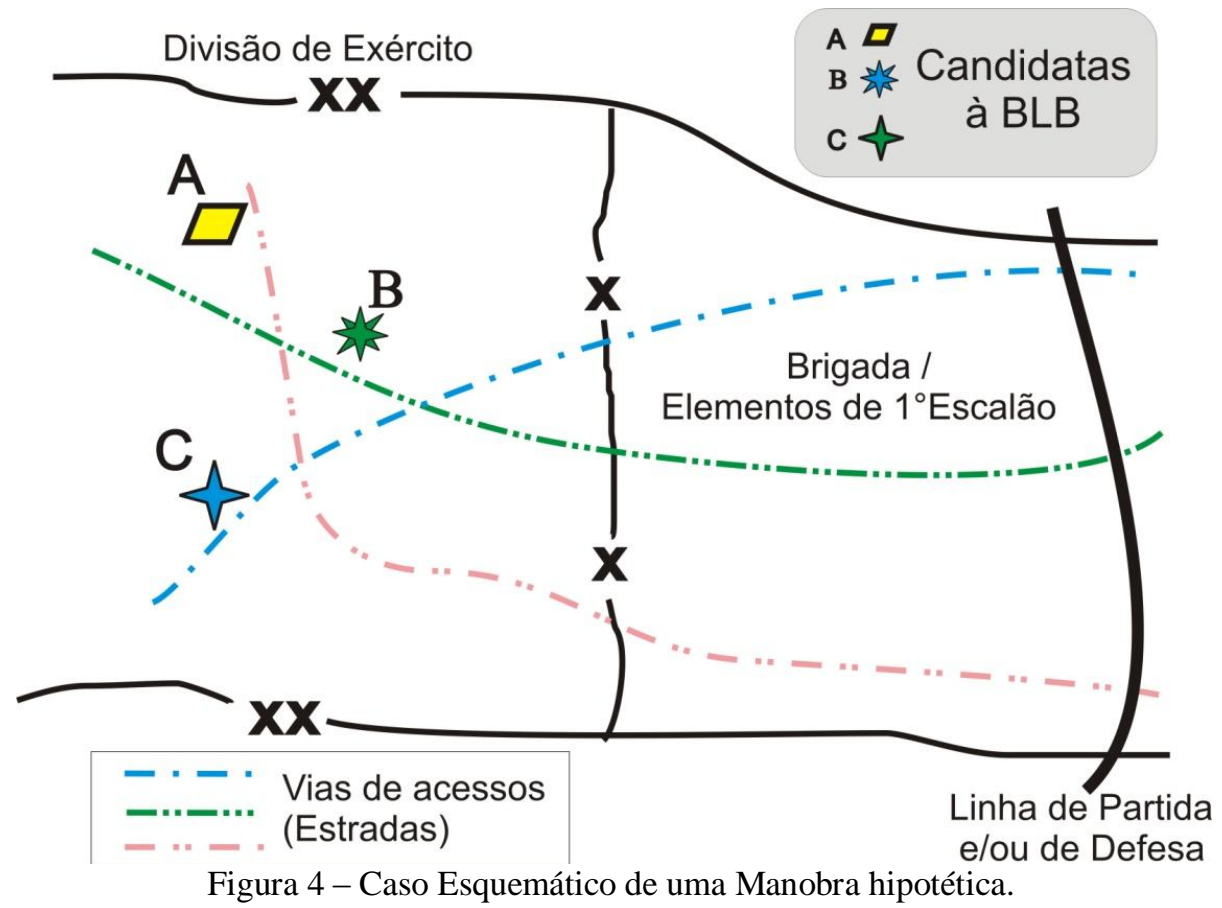

\subsection{Definição dos Tomadores de Decisão e Analistas de Decisão}

Os tomadores de decisão serão os membros do Estado Maior (EM) de Organização Militar (OM) valor Divisão de Exército/Brigada e o analista de decisão é um o próprio autor deste documento e oficiais e logística do Exército Brasileiro.

\subsection{Análise e Estruturação do Problema Decisório}

Para clareza do trabalho, os membros do EM tem noção do processo decisório proposto e do tipo de resultado que será fornecido, para enfim servir de base e apoio a decisão final.

Por outro lado, os membros do EM, em especial o Comandante Tático, o Oficial de Operações (E3) e o Oficial de Logística (E4) fornecem detalhes da operação a fim de proporcionar aos analistas reajustes finos no algoritmo a ser executado. Com isso, o problema tende a ficar mais claro para tomadores de decisão e para os analistas de decisão, dando mais transparência e objetividade para o processo de avaliação. 


\subsection{Definição de Categorias e Funções de Contribuições}

Para este problema foram definidas 4 (quatro) categorias de avaliação a saber: Excelente(E), Muito Adequado (MA), Adequado (A), Pouco Adequado (PA). Assim a partir dessas categorias são definidas as contribuições que serão dadas a cada um dos critérios.

O critério Manobra (Distância Máxima de Apoio - DMA) será tratado como quantitativo, pois será pontuado de acordo com as distâncias estimadas da AApLog até a ATE/ATC das OM apoiadas. Para isso serão definidas para este critério uma função de contribuição utilizando funções trapezoidais conforme mostrado na Figura 5.

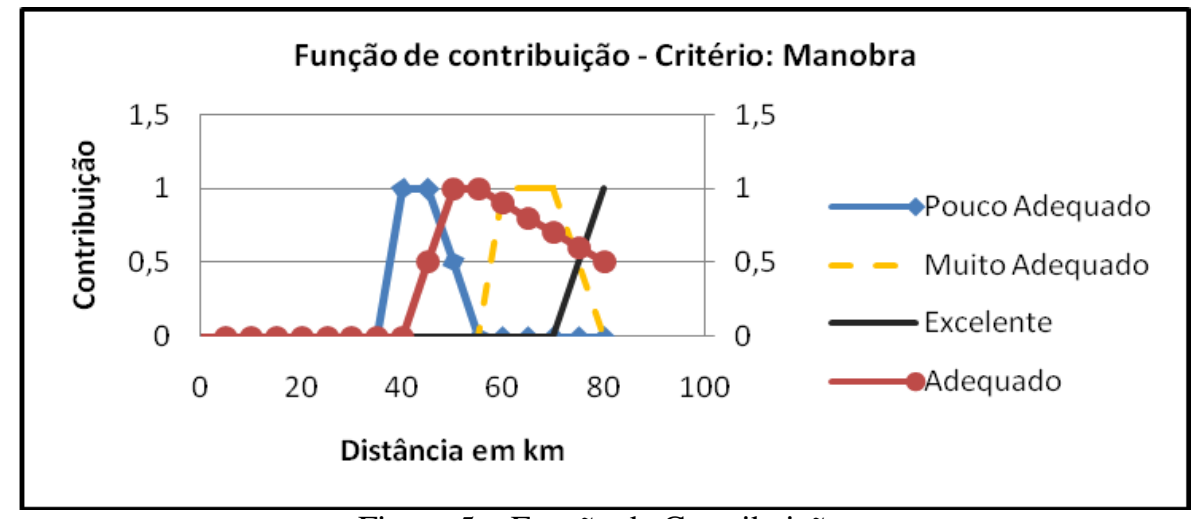

Figura 5 - Função de Contribuição.

O critério Terreno (Rede Rodoviária), Segurança (Segurança das Instalações) e Situação Logística (Estrada Principal de Suprimento - EPS) serão tratados como critérios qualitativos e por isso serão definidos para eles uma tabela de contribuições conforme Tabelas $4 ; 5$ e 6 .

\begin{tabular}{ccccc}
\hline \multirow{2}{*}{ Escala de Avaliação } & Excelente (E) & Muito adequado (MA) & Adequado (A) & $\begin{array}{c}\text { Pouco } \\
\text { Adequado (PA) }\end{array}$ \\
\hline Capacidade de Tráfego excelente & 1,0 & 0,9 & 0,5 & 0,0 \\
Capacidade de Tráfego normal & 0,5 & 0,7 & 1,0 & 0,2 \\
Capacidade de Tráfego deficiente & 0,2 & 0,5 & 0,7 & 1,0 \\
\hline
\end{tabular}

Tabela 4: Tabela de Contribuições para o critério Terreno; Partindo das análises dos oficiais auxiliares do EM especial é possível identificar, avaliar se a área atende aos quesitos/fatores para seleção.

\begin{tabular}{ccccc}
\hline Escala de Avaliação & Excelente (E) & $\begin{array}{c}\text { Muito adequado } \\
\text { (MA) }\end{array}$ & Adequado (A) & $\begin{array}{c}\text { Pouco } \\
\text { Adequado (PA) }\end{array}$ \\
\hline Permite Grande Dispersão & 1,0 & 0,9 & 0,8 & 0,5 \\
Permite Média Dispersão & 0,3 & 0,6 & 1,0 & 0,4 \\
Permite Pequena Dispersão & 0,1 & 0,3 & 0,7 & 1,0 \\
Não permite Dispersão & 0,0 & 0,1 & 0,5 & 1,0 \\
\hline
\end{tabular}

Tabela 5: Tabela de Contribuições para o critério Segurança; Partindo das análises dos oficiais auxiliares do EM especial é possível identificar, avaliar se a área atende aos quesitos/fatores para seleção.

\begin{tabular}{ccccc}
\hline Escala de Avaliação & Excelente (E) & $\begin{array}{c}\text { Muituação Logística - Categorias } \\
\text { (MA) }\end{array}$ & Adequado (A) & $\begin{array}{c}\text { Pouco } \\
\text { Adequado (PA) }\end{array}$ \\
\hline $\begin{array}{c}\text { Condição de Conservação } \\
\text { Ótima }\end{array}$ & 1,0 & 0,8 & 0,5 & 0,3 \\
$\begin{array}{c}\text { Condição de Conservação } \\
\text { Normal }\end{array}$ & 0,8 & 0,9 & 1,0 & 0,2 \\
$\begin{array}{c}\text { Condição de Conservação } \\
\text { Precária }\end{array}$ & 0,1 & 0,5 & 0,6 & 1,0 \\
\hline
\end{tabular}

Tabela 6: Tabela de Contribuições para o critério Situação Logística; Partindo das análises dos oficiais auxiliares do EM especial é possível identificar, avaliar se a área atende aos quesitos/fatores para seleção. 


\subsection{Definição da Importância Relativa entre os Critérios}

Conforme Passos et al (2013), a importância relativa entre os critérios será definida da mesma forma que no método TODIM. Primeiramente é montada uma matriz de comparações paritárias com cada um dos critérios: Manobra (MAN); Terreno (TER); Segurança (SEG) e Situação Logística (SL).

Ela será preenchida utilizando a escala fundamental de Saaty (2006) descrita na Tabela 3. A Tabela 7 mostra a matriz de comparações paritárias preenchida. A partir desta matriz da Tabela 7 é obtido um novo vetor de pesos. Cada uma de suas componentes é calcula através da média aritmética das linhas da matriz citada.

\begin{tabular}{ccccc}
\hline & MAN & TER & SEG & SL \\
\hline MAN & 1 & 3 & 5 & 2 \\
TER & $1 / 3$ & 1 & 3 & 7 \\
SEG & $1 / 5$ & $1 / 3$ & 1 & 1 \\
SL & $1 / 2$ & $1 / 7$ & 1 & 1 \\
\hline
\end{tabular}

Tabela 7: Matriz de Comparações paritárias preenchidas utilizando a Escala fundamental de Saaty.

Depois disso é feita a normalização de cada um desses valores dividindo-os pela soma dessas médias. O resultado é o vetor de pesos $W$ cuja soma das componentes é igual a 1(um).

$$
W=\left[\begin{array}{llll}
0,3999 & 0,4120 & 0,0921 & 0,0961
\end{array}\right], \text { sendo que: } \quad \sum_{i=1}^{n} \mathrm{w}_{i}=1
$$

\subsection{Classificação de Cada Alternativa em Uma das Categorias Proposta}

Para efetuar a avaliação é necessário obter as características e dados das possíveis candidatas às áreas de apoio logístico, no caso em estudo serão utilizadas as candidatas à BLB ALFA, BRAVO e CHARLIE.

Com tais informações será possível obter os dados de entrada para a classificação usando o TODIM-FSE, seguindo as quantificações e qualificações contidas na Tabela 8.

\begin{tabular}{|c|c|c|c|}
\hline \multirow{2}{*}{ Critérios } & \multicolumn{3}{|c|}{ Avaliações da BLB } \\
\hline & ALFA & BRAVO & CHARLIE \\
\hline $\begin{array}{c}\text { Manobra } \\
\text { (DMA) }\end{array}$ & $73 \mathrm{~km}$ & $65 \mathrm{~km}$ & $75 \mathrm{~km}$ \\
\hline Terreno & Capacidade de & Capacidade de & Capacidade de \\
\hline (Rede Rodoviária) & tráfego excelente & tráfego deficiente & tráfego normal \\
\hline $\begin{array}{c}\text { Segurança } \\
\text { (Segurança das Instalações) }\end{array}$ & $\begin{array}{l}\text { Permite grande } \\
\text { dispersão }\end{array}$ & $\begin{array}{l}\text { Permite pequena } \\
\text { dispersão }\end{array}$ & $\begin{array}{l}\text { Permite grande } \\
\text { dispersão }\end{array}$ \\
\hline $\begin{array}{l}\text { Situação Logística } \\
\text { (EPS) }\end{array}$ & $\begin{array}{c}\text { Condiçãa de } \\
\text { conservação ótima }\end{array}$ & $\begin{array}{c}\text { Condição de } \\
\text { conservação precária }\end{array}$ & $\begin{array}{c}\text { Condição de } \\
\text { conservação precária }\end{array}$ \\
\hline
\end{tabular}

Tabela 8: Resultado das avaliações realizadas pelos oficiais e especialistas integrantes do EM.

Utilizando-se os dados das Tabelas 4, 5 e 6, de contribuições agrupadas dos critérios, e os dados obtidos no gráfico da Figura 5, determinam-se os valores da Tabela 9.

\begin{tabular}{c|cccc|cccc|cccc}
\hline & \multicolumn{4}{|c|}{ BLB - ALFA } & \multicolumn{4}{c|}{ BLB - BRAVO } & \multicolumn{4}{c}{ BLB-CHARLIE } \\
Critérios & E & MA & A & PA & E & MA & A & PA & E & MA & A & PA \\
\hline $\begin{array}{c}\text { Manobra (DMA) } \\
\text { Terreno }\end{array}$ & 0,20 & 1,00 & 0,65 & 0,00 & 0,00 & 1,00 & 0,70 & 0,00 & 0,50 & 0,50 & 0,60 & 0,00 \\
(Rede Rodoviária) & 1,00 & 0,90 & 0,50 & 0,00 & 0,20 & 0,50 & 0,70 & 1,00 & 0,50 & 0,70 & 1,00 & 0,20 \\
$\begin{array}{c}\text { Segurança } \\
\text { Segurança das Instalações) }\end{array}$ & 1,00 & 0,90 & 0,80 & 0,50 & 0,10 & 0,30 & 0,70 & 1,00 & 1,00 & 0,90 & 0,80 & 0,50 \\
Situação Logística(EPS) & 1,00 & 0,80 & 0,50 & 0,30 & 0,10 & 0,50 & 0,60 & 1,00 & 0,10 & 0,50 & 0,60 & 1,00 \\
\hline
\end{tabular}

Tabela 9: Tabela de contribuições agrupadas dos critérios Resultado das avaliações realizadas pelos oficiais e especialistas integrantes do EM. 
Após realizar a aplicação da Fase 6 do item 3, chega-se ao resultado das alternativas normalizadas pela última equação do método TODIM, conforme a Tabela 10.

\begin{tabular}{ccccc}
\hline $\begin{array}{c}\text { Base Logística Brigada (BLB) } \\
\text { ou }\end{array}$ & Excelente & Muito Adequada & Adequada & Pouco Adequada \\
AApLog & & & & \\
\hline ALFA & 1,0000 & 0,9979 & 0,6437 & 0,0000 \\
BRAVO & 0,0000 & 0,6354 & 0,8591 & 1,0000 \\
CHARLIE & 0,4138 & 0,7882 & 1,0000 & 0,0000 \\
\hline
\end{tabular}

Tabela 10 - Resultado final com utilização do método TODIM-FSE.

\section{DISCUSSÕES E CONCLUSÕES}

A utilização do método TODIM-FSE evidenciou uma avaliação de BLB com o estudo de um caso hipotético. O objetivo desta avaliação foi dar suporte à seleção da melhor área apropriada segundo os diversos critérios e subcritérios para um desdobramento de tropas de apoio logístico, baseado na Doutrina Militar da Força Terrestre Brasileira.

Utilizando as informações obteve-se como resultado os valores apresentados na Tabela 10, que qualifica as alternativas "ALFA", "BRAVO" e "CHARLIE" respectivamente nas categorias: excelente, pouco adequada e adequada.

O método TODIM-FSE é uma metodologia de apoio à decisão multicritério de classificação de alternativas discretas que apresenta resultado satisfatório quando comparado com a análise realizada pelos tomadores de decisão.

A estrutura e as modalidades de aplicação foram completamente descritos. Um estudo de caso hipotético ilustrou como o modelo construído pode ser posto em prática. Usando estes métodos as categorias são construídas experimentalmente, em conjunto com os decisores, depois da construção do modelo. As fronteiras para cada categoria irá depender de um conjunto de alternativas previamente definidas, ou mesmo em dados fictícios, utilizados pelos decisores como referências.

Geralmente, uma grande quantidade de informações são necessárias para construir as categorias dentro destas metodologias. Conforme Passos et al (2013), outra característica marcante do TODIM -FSE está relacionado à possibilidade de fusão de elementos de lógica fuzzy e Teoria da Perspectiva. Desta forma, é possível considerar a imprecisão, geralmente presente em julgamentos humanos. Também é importante ressaltar o fato de que o conhecimento da lógica fuzzy é necessário para construir os próprios modelos, no entanto, este mesmo conhecimento não é necessário para realmente usar os modelos. Assim, uma vez que os modelos são construídos por um analista de decisão, os usuários irão incorporar as suas preferências por meio de escalas, sem qualquer característica difusa. Esta vantagem, sem dúvida, contribui para a relativa facilidade de usar o método TODIM-FSE.

O uso da Teoria dos Prospectos, incorporado nas equações TODIM, permite aos tomadores de decisão, no caso em estudo - os Oficiais Comandantes, a possibilidade de considerar o risco no problema de decisão. Isto é completamente adequado e desejável para a situação de emprego da tropa.

Assim, o método TODIM-FSE, aplicado no caso, apresentou resposta compatível para classificação de uma base logística. Portanto, a aplicação do método sugere ser adequada no sentido de auxiliar os analistas militares em aperfeiçoar a tomada de decisão visando à definição da Base Logística de Brigada mais coerente dentro da Zona de Combate.

\section{REFERÊNCIAS BIBLIOGRÁFICAS}

[1] Bana e Costa, C.A.; Vansnick, J.C., A critical analysis of the eigenvalue method used to derive priorities in AHP, European Journal Operational Research (2008). 
[2] Belton, V.; Stewart, T. Multiple Criteria Decision Analysis: An Integrated Approach (2002).

[3] Chang, N.B.; Chen, H.W.; Ning, S.K, Identification of river quality water quality using the fuzzy synthetic evaluation approach. Journal Environment Management (2001).

[4] Goodwin, P.; Wright, G., Decision Analysis for Management Judgment. Chichester: John Wiley and Sons (2004).

[5] Gomes, L. F. A. M., Tomada de decisões em cenários complexos. São Paulo: Thomson Learning (2004).

[6] Gomes, L.F.A.M.; Rangel, L.A.D.; Maranhão, F.J.C., Multi-criteria analysis of natural gas destination in Brazil: an application of the TODIM method. Mathematical Computer Model (2009).

[7] Gomes, L.F.A.M.; Rangel, L.A.D., An application of the TODIM method to the multi-criteria rental evaluation of residential properties. European Journal Operational Research (2009).

[8] Gomes, L.F.A.M.; Lima, M.M.P.P., From modeling individual preferences to multicritério ranking of discrete alternatives: a look at prospect theory and the additive difference model. Foundations of Computing and Decision Sciences (1992).

[9] Kahneman, D.; Tversky, A., Prospect theory: An analysis of decision under risk. Econometrica (1979).

[10] Keeney, RL.; Raiffa, H., Decision with multiples objectives: preferences and value tradeoffs. New York: Wiley (1976).

[11] Kuo, Y.F.; Chen, P.C., Selection of mobile value-added services for system operators using fuzzy synthetic evaluation. Expert Systems with Applications (2006).

[12] Lu, R.S.; Lo, S.L.; Hu, J.Y., Analysis of reservoir water quality using fuzzy synthetic evaluation. Stoch Environ Res Risk Assessement (1999).

[13] Ministério da Defesa (MD), Manual de Campanha EB20-MC-10.204 - Logística. Estado-Maior do Exército, 3.ed (2014).

[14] Ministério da Defesa, Manual de Campanha - Apoio Logístico na Divisão de Exército e na Brigada. Estado-Maior do Exército (2003) .

[15] Onkal-Engin, G; Demir, I., Assessment of urban air quality in Istanbul using fuzzy synthetic evaluation. Atmospheric Environment (2004).

[16] Passos A.C.; Teixeira M.G.; Garcia K.C.; Cardoso, A.M.; Gomes, L.F.A.M., Using the TODIM-FSE method as a decision-making support methodology for oil spill response. Computers and Operations Research (2013).

[17] Rangel, LAD, Gomes, LFAM, Cardoso, FP. An application of the TODIM method to the evaluation of broadband Internet plans. Pesquisa Operacional (2011).

[18] Saaty, T.L., Fundamentals of decision making and priority theory with the analytic hierarchy process. Pittsburgh: RWS Publishing (2006).

[19] Sadiq, R; Husain, T; Veitch, B; Bose, N., Risk-based decision-making for drilling waste discharges using a fuzzy synthetic evaluation technique. Ocean Engineering (2004). 